\title{
A Near-Optimal Decentralized Servomechanism Controller for Hierarchical Interconnected Systems
}

\author{
Javad Lavaei, Ahmadreza Momeni and Amir G. Aghdam
}

\begin{abstract}
This paper is concerned with decentralized output regulation of hierarchical systems subject to input and output disturbances. It is assumed that the disturbance can be represented as the output of an autonomous LTI system with unknown initial state. The primary objective is to design a decentralized controller with the property that not only does it reject the degrading effect of the disturbance on the output (for a satisfactory steady-state performance), it also results in a small LQ cost function (implying a good transient behavior). To this end, the underlying problem is treated in two phases. In the first step, a number of modified systems are defined in terms of the original system. The problem of designing a LQ centralized controller which stabilizes all the modified systems and rejects the disturbance in the original system is considered, and it is shown that this centralized controller can be efficiently found by solving a LMI problem. In the second step, a method recently presented in the literature is exploited to decentralize the designed centralized controller. It is proved that the obtained controller satisfies the pre-determined design specifications including disturbance rejection. Simulation results elucidate the efficacy of the proposed control law.
\end{abstract}

\section{INTRODUCTION}

In the control literature, an interconnected system is often referred to a system with a collection of interacting subsystems [1]. In terms of the interaction topology between the subsystems, the class of hierarchical interconnected systems has drawn special attention in recent publications due to its broad applications such as formation flying, underwater vehicles, automated highway, robotics, satellite constellation, etc., which have leader-follower structures or structures with virtual leaders [2], [3], [4], [5], [6]. It is shown in [2] that even if a continuous-time interconnected system does not have a hierarchical structure, under certain conditions its discrete-time equivalent model can be transformed to a hierarchical form. For such a system, it is normally desired to design a set of local controllers corresponding to the individual subsystems, which partially exchange their information [4], [7]. This demand is originated from some practical limitations concerning, for instance, the geographical distribution of the subsystems or the computational complexity associated with a centralized controller [8]. The case when

This work has been supported by the Natural Sciences and Engineering Research Council of Canada under grant RGPIN-262127-07.

Javad Lavaei is with the Department of Control and Dynamical Systems, California Institute of Technology, Pasadena, USA (email: lavaei@cds.caltech.edu)

Ahmadreza Momeni is with the Department of Electrical and Computer Engineering, Concordia University, Montreal, Canada (email: a_momeni@ece.concordia.ca).

Amir G. Aghdam is with the Department of Electrical and Computer Engineering, Concordia University, Montreal, Canada (email: aghdam@ece.concordia.ca). these local controllers operate independently (i.e., they do not interact with each other), is referred to as decentralized feedback control [9], [10], [11].

Various aspects of the decentralized control theory have been extensively investigated in the past few decades. The papers [10], [12], [13], [14] study the decentralized stabilizability of a system by using the notions of decentralized fixed modes and quotient fixed modes. Several approaches are proposed in the literature to solve the pole-placement problem by means of decentralized controllers [15], [16]. Furthermore, high-performance decentralized control design techniques have been investigated in [17], [18].

Since the real-world systems are usually vulnerable to external disturbances, the controller being designed for a hierarchical interconnected system is desired to satisfy the following properties:

i) The disturbances must be rejected in the steady state.

ii) A predefined $\mathrm{H}_{2}$ performance index should be minimized to achieve a fast transient response with an acceptable control energy.

iii) The controller to be designed should be decentralized. There exist a number of works which have addressed the problem of designing a controller satisfying the properties (i) and (iii) given above, and the controller obtained is regarded as decentralized servomechanism controller [19], [20], [21]. The paper [19] parameterizes all the decentralized controllers which reject the unmeasurable disturbances with known dynamics.

Moreover, the design of a controller which meets the criteria (ii) and (iii) has been studied intensively in several papers. In contrast to the $\mathrm{H}_{2}$ optimal centralized controller which can be simply obtained from the Riccati equation, the $\mathrm{H}_{2}$ optimal decentralized control problem involves sophisticated differential/nonconvex matrix equations [22], [23]. As a result, the available techniques often seek a nearoptimal solution, rather than a globally optimal one. For instance, a method is proposed in [24], which cuts off all the interconnections between the subsystems and designs local optimal controllers for the isolated subsystems accordingly. The main shortcoming of this approach is that the controller obtained may destabilize the system, in light of neglecting the system's interconnection parameters in the controller design. Another technique for handling the underlying problem is to impose the stringent constraint of staticness on the decentralized controller to be designed [25], [26]. More recently, a method is provided in [8] which decentralizes any given centralized controller of desired performance. As a byproduct of the results in [8], it is shown that the decentralized 
version of the $H_{2}$ optimal centralized controller is a $H_{2}$ nearoptimal decentralized controller. The only requirement of this approach is that the nominal model of the system is known by all control agents; i.e., every local controller must have a belief about the model of the entire system. This idea is further developed in [11] for the flight formation problem in the model predictive control framework. The paper [27], on the other hand, aims to design a controller for which all the aforementioned criteria (i), (ii) and (iii) hold. Since a set of nonlinear equations are derived in [27] for control design, this work cannot tackle the problem in question efficiently.

This paper presents a novel design strategy to obtain a high-performance decentralized control law for hierarchical interconnected systems, which is able to attenuate the effects of unmeasurable external disturbances with known dynamics. It is assumed that the state of each subsystem is available in its corresponding local output (this is not an unrealistic assumption in many applications such as vehicle formation problems [28]), and that the modeling parameters of the whole system are available (with some error) in any local station. It is to be noted that once a centralized controller is designed to achieve the properties (i) and (ii), its decentralized version (as can be obtained from [8]) does not necessarily maintain the same properties. To bypass this hurdle, a centralized controller is first designed to satisfy some artificial constraints (inspired by the conditions given in [11]). This controller is formulated in terms of LMI and can be obtained straightforwardly. The centralized controller elicited from the LMI problem is subsequently decentralized via the approach presented in [8]. Since the knowledge of each local controller about the whole system is inexact in practice, a procedure is proposed to measure the closeness of the designed decentralized controller to the optimal one in terms of the statistical information on the parameter deviation.

\section{Problem FORMULATION}

Consider a hierarchical interconnected system $\mathcal{S}$, whose $i^{\text {th }}$ subsystem $\mathcal{S}_{i}, i \in \bar{\nu}:=\{1,2, \ldots, \nu\}$, is represented by:

$$
\begin{aligned}
& \dot{x}_{i}(t)=\sum_{j=1}^{i} A_{i j} x_{j}(t)+B_{i} u_{i}(t)+E_{i} \omega(t) \\
& y_{i}(t)=C_{i} x_{i}(t)
\end{aligned}
$$

where $x_{i} \in \Re^{n_{i}}$ and $u_{i} \in \Re^{m_{i}}$ are the state and the input of the subsystem $\mathcal{S}_{i}$, respectively. Furthermore, $y_{i} \in \Re^{r_{i}}$ is the output of $\mathcal{S}_{i}$ to be regulated, and $\omega(t) \in \Re^{q}$ is the disturbance vector. Assume that the state $x_{i}(t)$ of the subsystem $\mathcal{S}_{i}$ is locally available, and that there is no measurement noise, i.e., the measured output in the $i^{\text {th }}$ subsystem is equal to $x_{i}(t)$. Suppose that the disturbance $\omega(t)$ can be expressed as:

$$
\begin{aligned}
\dot{z}(t) & =\Lambda z(t) \\
\omega(t) & =\mathcal{C} z(t)
\end{aligned}
$$

where the pair $(\mathcal{C}, \Lambda)$ is observable, and $z(0)$ is arbitrary and unknown.
The system $\mathcal{S}$ can be represented as follows:

$$
\begin{aligned}
\dot{x}(t) & =A x(t)+B u(t)+E \omega(t) \\
y(t) & =C x(t)
\end{aligned}
$$

where:

$$
\begin{aligned}
x(t) & =\left[\begin{array}{llll}
x_{1}(t)^{T} & x_{2}(t)^{T} & \cdots & x_{\nu}(t)^{T}
\end{array}\right]^{T}, \\
u(t) & =\left[\begin{array}{llll}
u_{1}(t)^{T} & u_{2}(t)^{T} & \cdots & x_{\nu}(t)^{T}
\end{array}\right]^{T} \\
y(t) & =\left[\begin{array}{llll}
y_{1}(t)^{T} & y_{2}(t)^{T} & \cdots & y_{\nu}(t)^{T}
\end{array}\right]^{T}, \\
E & =\left[\begin{array}{llll}
E_{1}^{T} & E_{2}^{T} & \cdots & E_{\nu}^{T}
\end{array}\right]^{T}, \\
B & =\operatorname{diag}\left(\left[\begin{array}{llll}
B_{1} & B_{2} & \cdots & B_{\nu}
\end{array}\right]\right), \\
C & =\operatorname{diag}\left(\left[\begin{array}{llll}
C_{1} & C_{2} & \cdots & C_{\nu}
\end{array}\right]\right)
\end{aligned}
$$

and $A$ is a $\nu \times \nu$ lower block triangular matrix whose $(i, j)$ block entry is equal to $A_{i j}$, for any $i, j \in \bar{\nu}, j \leq i$. Define now:

$$
n:=\sum_{i=1}^{\nu} n_{i}, \quad m:=\sum_{i=1}^{\nu} m_{i}, \quad r:=\sum_{i=1}^{\nu} r_{i}
$$

Suppose that the initial state $x(0)$ is a random variable with a given mean $X_{\mu}$ and variance $X_{\sigma}$. Define $X_{0}$ as:

$$
X_{0}:=\mathcal{E}\left\{x(0) x(0)^{T}\right\}=X_{\sigma}+X_{\mu} X_{\mu}^{T}
$$

where $\mathcal{E}\{\cdot\}$ represents the expectation operator. Furthermore, assume that the elements of the matrix $E$ given in (3) are arbitrary and unknown. The objective of this paper is introduced in Problem 1 given below.

Problem 1: Design a decentralized LTI controller $K_{d}$ (with block diagonal information flow structure [10]), such that the following conditions hold:

i) The state $x(t)$ goes to zero as $t \rightarrow \infty$, provided $z(0)=$ 0 .

ii) The output $y(t)$ approaches zero as $t \rightarrow \infty$, regardless of the initial state $z(0)$.

iii) When $z(0)$ is a zero vector, the performance index $J$ corresponding to the closed-loop system is satisfactorily small, where:

$$
J:=\mathcal{E}\left\{\int_{0}^{\infty}\left(x(t)^{T} Q x(t)+u(t)^{T} R u(t)\right) d t\right\}
$$

and where $R \in \Re^{m \times m}$ and $Q \in \Re^{n \times n}$ are positive definite and positive semi-definite matrices, respectively.

It is to be noted that since $E$ is an unknown matrix and can take any arbitrary value, it is desired that the controller $K_{d}$ be independent of $E$. The results obtained can be easily extended to the tracking problem in the presence of nonzero reference input, provided it can be expressed similarly to (2), with all of its modes located in the closed left half plane. This inclusion can be carried out by defining an augmented system and converting the tracking problem for the original system to a regulation one for the augmented system [29]. The following assumption is made without loss of generality.

Assumption 1: The matrices $B_{i}, C_{i}$ and $\mathcal{C}$ satisfy the following relations:

$\operatorname{rank}(\mathcal{C})=q, \quad \operatorname{rank}\left(B_{i}\right)=m_{i}, \quad \operatorname{rank}\left(C_{i}\right)=r_{i}, \quad i \in \bar{\nu}$ 
One can easily deduce from the results of [19] that the following two assumptions are required for the existence of the desired controller $\mathcal{K}_{d}$ under Assumption 1.

Assumption 2: The matrices given below are all full-rank:

$$
\left[\begin{array}{cc}
A_{i i}-\lambda_{j} I & B_{i} \\
C_{i} & 0
\end{array}\right], \quad \forall i \in \bar{\nu} \quad \text { and } \quad \forall j \in\{1,2, \ldots, p\}
$$

where $\lambda_{1}, \lambda_{2}, \ldots, \lambda_{p}$ denote the eigenvalues of $\Lambda$. Furthermore, the inequality $m_{i} \geq r_{i}$ holds for all $i \in \bar{\nu}$.

Assumption 3: The pair $\left(A_{i i}, B_{i}\right)$ is stabilizable for all $i \in \bar{\nu}$.

\section{Preliminaries}

In this section, it is desired to present the gist of the decentralization procedure given in [8]. Assume for now that $z(0)=0$, i.e., the system $\mathcal{S}$ is disturbance free. Define $x^{i}(t)$ and $u^{i}(t)$ as the vectors obtained from $x(t)$ and $u(t)$, respectively, by eliminating their block entries $x_{i}(t)$ and $u_{i}(t)$, for any $i \in \bar{\nu}$. Consider an arbitrary centralized LTI controller $K_{c}$ with the following state-space representation:

$$
\begin{aligned}
\dot{\eta}_{c}(t) & =\Gamma \eta_{c}(t)+\Omega x(t) \\
u(t) & =M \eta_{c}(t)+N x(t)
\end{aligned}
$$

where $\eta_{c} \in \Re^{\mu}$. There exist constant matrices $\boldsymbol{\Omega}^{i}, \boldsymbol{\Omega}_{i}, \mathbf{M}^{i}, \mathbf{M}_{i}, \mathbf{N}^{i}, \mathbf{N}_{i}, \mathbf{N}^{\bar{i}}$ and $\mathbf{N}_{\bar{i}}$, such that the above controller can be expressed in a decomposed representation as follows:

$$
\begin{aligned}
\dot{\eta}_{c}(t) & =\Gamma \eta_{c}(t)+\mathbf{\Omega}^{i} x^{i}(t)+\boldsymbol{\Omega}_{i} x_{i}(t) \\
u^{i}(t) & =\mathbf{M}^{i} \eta_{c}(t)+\mathbf{N}^{i} x^{i}(t)+\mathbf{N}_{i} x_{i}(t) \\
u_{i}(t) & =\mathbf{M}_{i} \eta_{c}(t)+\mathbf{N}^{\bar{i}} x^{i}(t)+\mathbf{N}_{\bar{i}} x_{i}(t)
\end{aligned}
$$

for any $i \in \bar{\nu}$. Similarly, there exist matrices $\mathbf{A}^{i}, \mathbf{A}^{\bar{i}}, \mathbf{A}_{i}$ and $\mathbf{B}^{i}$ (derived from $A$ and $B$ ) such that the system $\mathcal{S}$ given in (1) can be decomposed as follows:

$$
\begin{aligned}
& \dot{x}^{i}(t)=\mathbf{A}^{i} x^{i}(t)+\mathbf{A}_{i} x_{i}(t)+\mathbf{B}^{i} u^{i}(t) \\
& \dot{x}_{i}(t)=\mathbf{A}^{\bar{i}} x^{i}(t)+A_{i i} x_{i}(t)+B_{i} u_{i}(t)
\end{aligned}
$$

for any $i \in \bar{\nu}$. Define $K_{d_{i}}$ as a local controller for the subsystem $\mathcal{S}_{i}, i \in \bar{\nu}$, with the following state-space representation:

$$
\begin{aligned}
\dot{\eta}_{d_{i}}(t) & =\left[\begin{array}{cc}
\mathbf{A}^{i}+\mathbf{B}^{i} \mathbf{N}^{i} & \mathbf{B}^{i} \mathbf{M}^{i} \\
\boldsymbol{\Omega}^{i} & \Gamma
\end{array}\right] \eta_{d_{i}}(t) \\
& +\left[\begin{array}{c}
\mathbf{A}_{i}+\mathbf{B}^{i} \mathbf{N}_{i} \\
\boldsymbol{\Omega}_{i}
\end{array}\right] x_{i}(t) \\
u_{i}(t) & =\left[\begin{array}{cc}
\mathbf{N}^{\bar{i}} & \mathbf{M}_{i}
\end{array}\right] \eta_{d_{i}}(t)+\mathbf{N}_{\bar{i}} x_{i}(t)
\end{aligned}
$$

Define also $K_{d}$ as a decentralized controller consisting of the local controllers $K_{d_{1}}, K_{d_{2}}, \ldots ., K_{d_{\nu}}$.

Theorem 1: [8] Assume that $x(0)$ is a known vector (as opposed to a random variable). The state and the input of the system $\mathcal{S}$ under the centralized controller $K_{c}$ are the same as those of the system $\mathcal{S}$ under the decentralized controller $K_{d}$, if the initial state of the local controller $K_{d_{i}}$ is chosen as:

$$
\eta_{d_{i}}(0)=\left[\begin{array}{c}
x^{i}(0) \\
0
\end{array}\right], \quad i \in \bar{\nu}
$$

Theorem 1 states that the centralized controller $K_{c}$ can be transformed to an equivalent decentralized controller $K_{d}$, if the initial state $x(0)$ is a known vector and any local controller $K_{d_{i}}, i \in \bar{\nu}$, exactly knows the initial states of the other subsystems. It is to be noted that these are not realistic assumptions in practice, and thus the result of Theorem 1 cannot be applied to the real-world problems. However, this result will be used later for the development of the main results of the paper, where the practical limitations are taken into account. As the first step, assume that $x(0)$ is only statistically known, and hence let the following initial state be deployed:

$$
\eta_{d_{i}}(0)=\left[\begin{array}{c}
X_{\mu}^{i} \\
0
\end{array}\right], \quad i \in \bar{\nu}
$$

instead of the one in (14). In the sequel, the internal stability of the system $\mathcal{S}$ under the decentralized controller $K_{d}$ will be investigated.

Definition 1: Consider the system $\mathcal{S}$ given by (1). The modified system $\mathbf{S}^{i}, i \in \bar{\nu}$, is defined to be a system obtained by removing all interconnections going to the $i$ 'th subsystem in $\mathcal{S}$. The state-space representation of the modified system $\mathbf{S}^{i}$ is as follows:

$$
\begin{aligned}
\dot{x}(t) & =\tilde{A}^{i} x(t)+B u(t) \\
y(t) & =C x(t)
\end{aligned}
$$

where $\tilde{A}^{i}$ is derived from $A$ by replacing the first $i-1$ block entries of its $i$ 'th block row with zeros. It is to be noted that $\mathbf{S}^{1}=\mathcal{S}$.

Definition 2: Define the isolated subsystem $\mathcal{S}_{i}, i \in \bar{\nu}$, as a system obtained from the subsystem $\mathcal{S}_{i}$ by eliminating all of its incoming interconnections.

Theorem 2: [11] The system $\mathcal{S}$ is internally stable under the controller $K_{d}$ if and only if the system $\mathbf{S}^{i}$ is stable under the controller $K_{c}$, for all $i \in \bar{\nu}$.

A centralized servomechanism controller will be given in the next section, which will be used later as a reference to obtain the desired decentralized controller.

\section{A REFERENCE CENTRALIZED SERVOMECHANISM CONTROLLER}

To avoid trivial cases, assume with no loss of generality that all of the eigenvalues of $\Lambda$ lie in the closed right-half plane. It can be concluded from Assumptions 1, 2 and 3, and the results of [30], that there exist three nonunique matrices $\mathcal{B}, \mathcal{M}$ and $\mathcal{N}$, such that any minimum order centralized controller satisfying the requirements (i) and (ii) of Problem 1 can be represented by:

$$
\begin{aligned}
\dot{\eta}_{c}(t) & =\mathcal{A} \eta_{c}(t)+\mathcal{B} y(t) \\
u(t) & =\mathcal{M} \eta_{c}(t)+\mathcal{N} x(t)
\end{aligned}
$$

where:

$$
\mathcal{A}:=\operatorname{diag}\left(\left[\begin{array}{llll}
\Lambda & \Lambda & \cdots & \Lambda
\end{array}\right]\right)
$$

and where $(\mathcal{A}, \mathcal{B})$ is controllable. The objective of this section is to solve the problem introduced below. 
Problem 2: Find the matrices $\mathcal{B}, \mathcal{M}$, and $\mathcal{N}$, so that the centralized controller given by (17) has the following properties:

i) It satisfies the criteria (i) and (ii) of Problem 1.

ii) It stabilizes all of the systems $\mathbf{S}^{2}, \ldots, \mathbf{S}^{\nu}$.

iii) $\mathcal{B}$ is a block diagonal matrix, and the dimension of its $i^{\text {th }}$ block entry is $r_{i} p \times r_{i}$, for any $i \in \bar{\nu}$.

The centralized controller satisfying the conditions of Problem 2 will be transformed to a decentralized controller in the next section. It is to be noted that the conditions (ii) and (iii) given above are required in the decentralization procedure, as will be shown subsequently.

Lemma 1: Problem 2 has a solution, if and only if there exist a block diagonal matrix $\mathcal{B}$, matrices $\mathcal{M}$ and $\mathcal{N}$, and positive definite matrices $P_{1}, P_{2}, \ldots, P_{\nu}$ with the following properties:

$$
\begin{aligned}
& {\left[\begin{array}{cc}
\tilde{A}^{i} & 0 \\
\mathcal{B} C & \mathcal{A}
\end{array}\right]^{T} P_{i}+P_{i}\left[\begin{array}{cc}
\tilde{A}^{i} & 0 \\
\mathcal{B} C & \mathcal{A}
\end{array}\right]+\left[\begin{array}{cc}
\mathcal{Q} & 0 \\
0 & 0
\end{array}\right]} \\
& -P_{i}\left[\begin{array}{c}
B \\
0
\end{array}\right] R^{-1}\left[\begin{array}{c}
B \\
0
\end{array}\right]^{T} P_{i} \\
& +\left(R^{-\frac{1}{2}}\left[\begin{array}{c}
B \\
0
\end{array}\right]^{T} P_{i}+R^{\frac{1}{2}}\left[\begin{array}{cc}
\mathcal{N} & \mathcal{M}
\end{array}\right]\right) \\
& \times\left(R^{-\frac{1}{2}}\left[\begin{array}{c}
B \\
0
\end{array}\right]^{T} P_{i}+R^{\frac{1}{2}}\left[\begin{array}{ll}
\mathcal{N} & \mathcal{M}
\end{array}\right]\right)<0, \quad i \in \bar{\nu}
\end{aligned}
$$

Proof: Substituting (17a) into (16) results in the augmented system given below:

$$
\left[\begin{array}{c}
\dot{x}(t) \\
\dot{\eta}_{c}(t)
\end{array}\right]=\left[\begin{array}{cc}
\tilde{A}^{i} & 0 \\
\mathcal{B} C & \mathcal{A}
\end{array}\right]\left[\begin{array}{c}
x(t) \\
\eta_{c}(t)
\end{array}\right]+\left[\begin{array}{c}
B \\
0
\end{array}\right] u(t), \quad i \in \bar{\nu}
$$

It is inferred from [30] that the desired controller exists, if and only if there exist a block diagonal matrix $\mathcal{B}$, and matrices $\mathcal{M}$ and $\mathcal{N}$ such that the static controller $u(t)=$ $\left[\begin{array}{ll}\mathcal{N} & \mathcal{M}\end{array}\right]\left[\begin{array}{c}x(t) \\ \eta_{c}(t)\end{array}\right]$ stabilizes all of the augmented systems given by (20). Moreover, it follows from [31] that this stabilizability problem is equivalent to the solvability of the matrix inequality problem given in (19).

Theorem 3: Problem 2 has a solution, if and only if there exist block diagonal matrices $\mathcal{B}$ and $W$, matrices $\mathcal{M}$ and $\mathcal{N}$, and positive definite matrices $P_{1}, P_{2}, \ldots, P_{\nu}, V_{1}, V_{2}, \ldots, V_{\nu}$, such that the following matrix inequality problem:

$$
\left[\begin{array}{cc}
\Phi_{i} & \bar{\Phi}_{i} \\
\bar{\Phi}_{i}^{T} & -I
\end{array}\right]<0, \quad i \in \bar{\nu}
$$

is feasible, where:

$$
\begin{aligned}
& \Phi_{i}=\left[\begin{array}{cc}
\tilde{A}^{i} & 0 \\
0 & \mathcal{A}
\end{array}\right]^{T} P_{i}+P_{i}\left[\begin{array}{cc}
\tilde{A}^{i} & 0 \\
0 & \mathcal{A}
\end{array}\right] \\
& +\left[\begin{array}{l}
I \\
0
\end{array}\right] C^{T}\left(W^{T} W-\mathcal{B}^{T} W-W^{T} \mathcal{B}\right) C\left[\begin{array}{ll}
I & 0
\end{array}\right] \\
& +V_{i} G V_{i}-P_{i} G V_{i}-V_{i} G P_{i}+\left[\begin{array}{cc}
Q & 0 \\
0 & 0
\end{array}\right], \quad i \in \bar{\nu}, \\
& \bar{\Phi}_{i}=\left[\left(R^{-\frac{1}{2}}\left[\begin{array}{c}
B \\
0
\end{array}\right]^{T} P_{i}+R^{\frac{1}{2}}\left[\begin{array}{ll}
\mathcal{N} & \mathcal{M}
\end{array}\right]\right)^{T}\right. \\
& \left.\left[\begin{array}{cc}
0 & 0 \\
\mathcal{B} C & 0
\end{array}\right]^{T}+P_{i}\right], \quad i \in \bar{\nu}, \\
& G=\left[\begin{array}{c}
B \\
0
\end{array}\right] R^{-1}\left[\begin{array}{c}
B \\
0
\end{array}\right]^{T}+I
\end{aligned}
$$

Proof of necessity: Assume that Problem 2 has a solution. It can be concluded from Lemma 1 that there exist a block diagonal matrix $\mathcal{B}$, matrices $\mathcal{M}$ and $\mathcal{N}$, and positive definite matrices $P_{1}, P_{2}, \ldots, P_{\nu}$, such that the matrix inequality problem given in (19) is feasible. One can easily verify that the matrix inequality problem (21) for the matrix variables $\mathcal{B}, \mathcal{M}, \mathcal{N}, W, P_{1}, \ldots, P_{\nu}, V_{1}, \ldots, V_{\nu}$, where $W=\mathcal{B}$ and $V_{i}=$ $P_{i}, \forall i \in \bar{\nu}$, is the same as the one expressed by (19).

Proof of sufficiency: Suppose that there exist block diagonal matrices $\mathcal{B}$ and $W$, matrices $\mathcal{M}$ and $\mathcal{N}$, and positive definite matrices $P_{1}, \ldots, P_{\nu}, V_{1}, \ldots, V_{\nu}$, such that the matrix inequality problem (21) is feasible. Applying the Schur complement's formula to (21), one can conclude that:

$$
\bar{\Phi}_{i} \bar{\Phi}_{i}^{T}+\Phi_{i}<0, \quad i \in \bar{\nu}
$$

On the other hand, it is known that:

$$
\left(P_{i}-V_{i}\right) G\left(P_{i}-V_{i}\right) \geq 0, \quad C^{T}(B-W)^{T}(B-W) C \geq 0
$$

The above inequalities are equivalent to the following ones:

$$
\begin{aligned}
& V_{i} G V_{i}-V_{i} G P_{i}-P_{i} G V_{i} \geq-P_{i} G P_{i} \\
& C^{T} W^{T} W C-C^{T} \mathcal{B}^{T} W C-C^{T} W^{T} \mathcal{B} C \geq-C^{T} \mathcal{B}^{T} \mathcal{B} C
\end{aligned}
$$

The inequalities (23), (25a) and (25b) lead to the following:

$$
\begin{aligned}
& \bar{\Phi}_{i} \bar{\Phi}_{i}^{T}+\left[\begin{array}{cc}
\tilde{A}^{i} & 0 \\
0 & \mathcal{A}
\end{array}\right]^{T} P_{i}+P_{i}\left[\begin{array}{cc}
\tilde{A}^{i} & 0 \\
0 & \mathcal{A}
\end{array}\right]-P_{i} G P_{i} \\
& -\left[\begin{array}{l}
I \\
0
\end{array}\right] C^{T} \mathcal{B}^{T} \mathcal{B} C\left[\begin{array}{ll}
I & 0
\end{array}\right]+\left[\begin{array}{cc}
Q & 0 \\
0 & 0
\end{array}\right]<0, i \in \bar{\nu}
\end{aligned}
$$

The proof follows from Lemma 1 and from the fact that the expressions in the left sides of the inequalities (19) and (26) are identical.

Remark 1: It can be easily verified that the matrix inequalities (21) turn to be LMIs when $V$ and $W$ are set to be constants.

Consider now the $i^{\text {th }}$ isolated subsystem $\mathcal{S}_{i}$ :

$$
\begin{aligned}
\dot{x}_{i}(t) & =A_{i i} x_{i}(t)+B_{i} u_{i}(t)+E_{i} \omega(t) \\
y_{i}(t) & =C_{i} x_{i}(t)
\end{aligned}
$$


Pursuing the method proposed in [30] and using Assumptions 1,2 and 3 , one can obtain the matrices $\mathcal{B}_{i}, \mathcal{M}_{i}$ and $\mathcal{N}_{i}$, for any $i \in \bar{\nu}$, such that the controller:

$$
\begin{aligned}
\dot{\eta}_{c_{i}}(t) & =\mathcal{A}_{i} \eta_{c_{i}}(t)+\mathcal{B}_{i} y_{i}(t) \\
u_{i}(t) & =\mathcal{M}_{i} \eta_{c_{i}}(t)+\mathcal{N}_{i} x_{i}(t)
\end{aligned}
$$

attenuates the state $x_{i}(0)$ of the system given in (27) to zero provided $z(0)=0$, and regulates $y_{i}(t)$ to zero for any arbitrary $z(0)$, where:

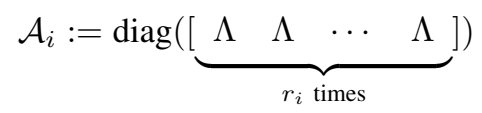

Define now the following matrices:

$$
\begin{aligned}
\mathcal{B}_{o} & =\operatorname{diag}\left(\left[\begin{array}{llll}
\mathcal{B}_{1} & \mathcal{B}_{2} & \cdots & \mathcal{B}_{\nu}
\end{array}\right]\right), \\
\mathcal{M}_{o} & =\operatorname{diag}\left(\left[\begin{array}{llll}
\mathcal{M}_{1} & \mathcal{M}_{2} & \cdots & \mathcal{M}_{\nu}
\end{array}\right]\right), \\
\mathcal{N}_{o} & =\operatorname{diag}\left(\left[\begin{array}{llll}
\mathcal{N}_{1} & \mathcal{N}_{2} & \cdots & \mathcal{N}_{\nu}
\end{array}\right]\right)
\end{aligned}
$$

By considering $\mathcal{B}=\mathcal{B}_{0}, \mathcal{M}=\mathcal{M}_{0}$, and $\mathcal{N}=\mathcal{N}_{0}$, it can be easily concluded that the controller (17) is a solution to Problem 2. Therefore, from Lemma 1 there exist positive definite matrices $P_{1}^{0}, \ldots, P_{\nu}^{0}$, such that the matrix inequalities (19) hold for $\mathcal{B}=\mathcal{B}_{0}, \mathcal{M}=\mathcal{M}_{0}, \mathcal{N}=\mathcal{N}_{0}$ and $P_{i}=P_{i}^{0}$, $\forall i \in \bar{\nu}$. It is to be noted that the quadratic terms with respect to $P_{i}$ in (19) are eliminated, which implies that (19) is a LMI with respect to $P_{i}$, and thus can be solved using the available LMI solvers.

An algorithm is introduced next, which aims to design a centralized controller solving Problem 2, while it meets the condition (iii) of Problem 1 as well.

\section{Algorithm 1:}

Step 1) Set $W=\mathcal{B}_{0}$ and $V_{i}=P_{i}^{0}$ for all $i \in \bar{\nu}$.

Step 2) Minimize the objective function $\operatorname{trace}\left(P_{1} X_{0}\right)$ for the variables $\mathcal{B}, \mathcal{M}, \mathcal{N}$ and $P_{1}, \ldots, P_{\nu} \geq 0$, subject to the inequality constraints (21), which are LMIs (according to Remark 1), and the constraint that $\mathcal{B}$ is block diagonal (note that $X_{0}$ is defined in (6)).

Step 3) If $\sum_{i=1}^{\nu}\left\|V_{i}-P_{i}\right\|+\|W-\mathcal{B}\| \leq \delta$, where $\delta$ is a prescribed permissible deviation, then stop. Otherwise, set $V_{i}=P_{i}, i \in \bar{\nu}$, and $W=\mathcal{B}$, and go to Step 2 .

Let the matrices $\mathcal{B}, \mathcal{M}$ and $\mathcal{N}$ obtained in Algorithm 1 be denoted by $\mathcal{B}_{\text {opt }}, \mathcal{M}_{\text {opt }}$ and $\mathcal{N}_{\text {opt }}$, respectively. It can be easily seen that the control (17) with the parameters $\mathcal{B}_{\text {opt }}$, $\mathcal{M}_{\text {opt }}$ and $\mathcal{N}_{\text {opt }}$ satisfies the requirements of Problem 2 and the condition (iii) of Problem 1.

Remark 2: The objective function trace $\left(P_{1} X_{0}\right)$ introduced in Step 2 of Algorithm 1 is, in fact, equivalent to the performance index $J$ given by (7). The details of this equivalency may be found in [32].

Remark 3: As pointed out earlier, the matrix inequalities given by (19) are satisfied for $\mathcal{B}=\mathcal{B}_{0}, \mathcal{M}=\mathcal{M}_{0}, \mathcal{N}=\mathcal{N}_{0}$, and $P_{i}=P_{i}^{0}, i \in \bar{\nu}$. On the other hand, by setting $V_{i}=P_{i}^{0}$ and $W=\mathcal{B}_{0}$, the LMIs (21) will be equivalent to the matrix inequalities (19). This implies that the LMI problem given in Step 2 of Algorithm 1 is feasible. In addition, it is evident that this algorithm is monotone decreasing and convergent, and should ideally stop when $W=\mathcal{B}$ and $V_{i}=P_{i}$ for all $i \in \bar{\nu}$. This results from the conditions under which the inequalities (25a) and (25b) turn to the equalities. However, Step 3 is required in order for the algorithm to halt in a finite number of iterations.

The centralized servomechanism controller obtained here will be used in the next section to find a high-performance decentralized servomechanism controller.

\section{OPTIMAL DECENTRALIZED SERVOMECHANISM CONTROLLER}

Consider the centralized controller $\tilde{K}_{c}$ of the form (10) with the following parameters:

$$
\begin{aligned}
\dot{\eta}_{c}(t) & =\mathcal{A} \eta_{c}(t)+\mathcal{B}_{\text {opt }} C x(t) \\
u(t) & =\mathcal{M}_{\text {opt }} \eta_{c}(t)+\mathcal{N}_{\text {opt }} x(t)
\end{aligned}
$$

The methodology proposed in Section III can now be applied to the centralized controller $\tilde{K}_{c}$ in order to obtain a decentralized controller denoted by $\tilde{K}_{d}$. For this purpose, let the above controller be decomposed as:

$$
\begin{aligned}
\dot{\eta}_{c}(t) & =\mathcal{A} \eta_{c}(t)+\mathbf{B}_{o p t}^{i} \mathbf{C}^{i} x^{i}(t)+\mathbf{B}_{i}^{o p t} C_{i} x_{i}(t) \\
u^{i}(t) & =\mathbf{M}_{o p t}^{i} \eta_{c}(t)+\mathbf{N}_{o p t}^{i} x^{i}(t)+\mathbf{N}_{i}^{o p t} x_{i}(t) \\
u_{i}(t) & =\mathbf{M}_{i}^{o p t} \eta_{c}(t)+\mathbf{N}_{o p t}^{i} x^{i}(t)+\mathbf{N}_{\bar{i}}^{o p t} x_{i}(t)
\end{aligned}
$$

where the matrices $\mathbf{C}^{i}, \mathbf{B}_{\text {opt }}^{i}, \mathbf{B}_{i}^{\text {opt }}, \mathbf{M}_{\text {opt }}^{i}, \mathbf{M}_{i}^{\text {opt }}, \mathbf{N}_{\text {opt }}^{i}, \mathbf{N}_{i}^{\text {opt }}$, $\mathbf{N}_{\text {opt }}^{\bar{i}}$ and $\mathbf{N}_{\bar{i}}^{\text {opt }}$ are derived from $C, \mathcal{B}_{\text {opt }}, \mathcal{M}_{\text {opt }}$ and $\mathcal{N}_{\text {opt }}$. Therefore, the state-space representation of the local controller $\tilde{K}_{d_{i}}, i \in \bar{\nu}$, will be obtained as follows:

$$
\begin{aligned}
\dot{\eta}_{d_{i}}(t) & =\left[\begin{array}{cc}
\mathbf{A}^{i}+\mathbf{B}^{i} \mathbf{N}_{o p t}^{i} & \mathbf{B}^{i} \mathbf{M}_{o p t}^{i} \\
\mathbf{B}_{o p t}^{i} \mathbf{C}^{i} & \mathcal{A}
\end{array}\right] \eta_{d_{i}}(t) \\
& +\left[\begin{array}{c}
\mathbf{A}_{i}+\mathbf{B}^{i} \mathbf{N}_{i}^{o p t} \\
\mathbf{B}_{i}^{o p t} C_{i}
\end{array}\right] x_{i}(t) \\
u_{i}(t) & =\left[\begin{array}{ll}
\mathbf{N}_{o p t}^{\bar{i}} & \mathbf{M}_{i}^{\text {opt }}
\end{array}\right] \eta_{d_{i}}(t)+\mathbf{N}_{\bar{i}}^{o p t} x_{i}(t)
\end{aligned}
$$

Suppose that the initial state of the controller $\tilde{K}_{d_{i}}$ is equal to $\eta_{d_{i}}(0)=\left[\begin{array}{cc}X_{\mu}^{i^{T}} & 0_{1 \times r p}\end{array}\right]^{T}$, for all $i \in \bar{\nu}$, where $0_{1 \times r p}$ denotes the $1 \times r p$ zero matrix. It is desired to prove that $\tilde{K}_{d}$ is a solution of Problem 1.

Theorem 4: The decentralized controller $\tilde{K}_{d}$ satisfies the requirements (i) and (ii) of Problem 1 for the system $\mathcal{S}$.

Proof: Since $\tilde{K}_{c}$ given by (31) is designed in Section IV in such a way that it stabilizes the modified system $S^{i}$ for any $i \in \bar{\nu}$, it can be concluded from Theorem 2 that the state $x(t)$ of the system $\mathcal{S}$ under the decentralized controller $\tilde{K}_{d}$ goes to zero as $t \rightarrow \infty$, provided $z(0)=0$. Thus, the requirement (i) of Problem 1 is met. Denote the block diagonal matrix $\mathcal{B}_{\text {opt }}$ as:

$$
\mathcal{B}_{\text {opt }}=\operatorname{diag}\left(\left[\begin{array}{llll}
\mathcal{B}_{11}^{\text {opt }} & \mathcal{B}_{22}^{\text {opt }} & \cdots & \mathcal{B}_{\nu \nu}^{\text {opt }}
\end{array}\right]\right)
$$

It can be easily verified that $\mathbf{B}_{i}^{\text {opt }}$ introduced in (32) is equal to:

$$
\begin{aligned}
& \mathbf{B}_{i}^{\text {opt }}=\left[\begin{array}{llll}
0_{r_{i} \times r_{1} p} & \cdots & 0_{r_{i} \times r_{i-1} p} & \mathcal{B}_{i i}^{\text {opt }}{ }^{T}
\end{array}\right. \\
& \left.\begin{array}{lll}
0_{r_{i} \times r_{i+1} p} & \cdots & 0_{r_{i} \times r_{\nu} p}
\end{array}\right]^{T}, \quad i \in \bar{\nu}
\end{aligned}
$$


Furthermore, $\mathbf{B}_{\text {opt }}^{i} \mathbf{C}^{i}$ is derived from $\mathcal{B}_{\text {opt }} C$ by removing its $i^{\text {th }}$ block column (which is equal to $\mathbf{B}_{i}^{o p t} C_{i}$ ). This observation along with the fact that $\mathcal{B}_{\text {opt }}$ and $C$ are block diagonal, yield that the $i^{\text {th }}$ block row of $\mathbf{B}_{o p t}^{i} \mathbf{C}^{i}$ is a zero matrix. Using this result and substituting (35) into (33), one can rearrange the entries of the state vector $\eta_{d_{i}}(t)$ in order to come up with the following state-space representation for the local controller $\tilde{K}_{d_{i}}$ :

$$
\begin{aligned}
\dot{\tilde{\eta}}_{d_{i}}(t) & =\left[\begin{array}{cc}
\mathcal{A}_{i} & 0 \\
L_{i_{1}} & L_{i_{2}}
\end{array}\right] \tilde{\eta}_{d_{i}}(t) \\
& +\left[\begin{array}{cc}
\mathcal{B}_{i i}^{o p t} & 0 \\
0 & L_{i_{3}}
\end{array}\right]\left[\begin{array}{cc}
y_{i}(t) & 0 \\
0 & x_{i}(t)
\end{array}\right] \\
u_{i}(t) & =L_{i_{4}} \tilde{\eta}_{d_{i}}(t)+\mathbf{N}_{\bar{i}_{2}}^{\text {opt }} x_{i}(t)
\end{aligned}
$$

where $\mathcal{A}_{i}$ is defined in (29). Apply now the decentralized controller $\tilde{K}_{d}$ to the system $\mathcal{S}$. Each interconnection signal coming into the subsystem $\mathcal{S}_{i}$ from the other subsystems is composed of two main components: one is exponentially decaying (because the requirement (i) of Problem 1 is fulfilled) and hence does not affect the regulation of $y_{i}$, and the other one is an unbounded component whose effect is similar to $\omega(t)$ in (2). This unbounded component together with the disturbance term $E_{i} \omega(t)$ can be modeled in the statespace representation of the subsystem $\mathcal{S}_{i}$ as an embedded term $\tilde{E}_{i} \omega(t)$, where $\omega(t)$ is obtained from (2) with a proper initial condition $z(0)$. As a result, the $i^{\text {th }}$ subsystem can be modeled as:

$$
\begin{aligned}
\dot{x}_{i}(t) & =A_{i i} x_{i}(t)+B_{i} u_{i}(t)+G_{i} r_{i}(t)+\tilde{E}_{i} \omega(t) \\
y_{i}(t) & =C_{i} x_{i}(t)
\end{aligned}
$$

where $r_{i}(t)$ represents the exponentially decaying component of the incoming interconnections. Since the structure of $\tilde{K}_{d_{i}}$ in (36) complies with the controller proposed in [30], $y_{i}(t)$ approaches zero as $t \rightarrow \infty$, when the local controller $\tilde{K}_{d_{i}}$ (given by (36)) is applied to the system given by (37). This completes the proof.

So far, it is shown that the decentralized controller $\tilde{K}_{d}$ satisfies the requirements (i) and (ii) of Problem 1. The requirement (iii) will be investigated next.

Assume that the centralized controller $\tilde{K}_{c}$ is applied to the system $\mathcal{S}$. Denote the corresponding performance index (7) with $J_{o p t}$. Note that $J_{o p t}$ is derived from a constrained optimization problem, and ideally, it is desired to have the same performance for the decentralized control system. However, there is a deviation between the decentralized performance index and $J_{o p t}$. A method will be given next to measure this deviation.

The performance index $J$ associated with the system $\mathcal{S}$ under the decentralized controller $\tilde{K}_{d}$ can be written as $\operatorname{trace}\left(P_{d} X_{0}^{d}\right)$, where $P_{d}$ is derived from a Lyapunov equation [32], and:

$$
X_{0}^{d}=\left[\begin{array}{cccc}
X_{0} & X_{\mu} X_{\mu}^{1^{T}} & \cdots & X_{\mu} X_{\mu}^{\nu T} \\
X_{\mu}^{1} X_{\mu}^{T} & X_{\mu}^{1} X_{\mu}^{1 T} & \cdots & X_{\mu}^{1} X_{\mu}^{\nu T} \\
\vdots & \vdots & \ddots & \vdots \\
X_{\mu}^{\nu} X_{\mu}^{T} & X_{\mu}^{\nu} X_{\mu}^{1 T} & \cdots & X_{\mu}^{\nu} X_{\mu}^{\nu T}
\end{array}\right]
$$

According to Theorem 1 , if $X_{\mu}^{i}$ is equal to $x^{i}(0)$ for all $i \in \bar{\nu}$, then the state and the input of the centralized closed-loop system are the same as those of the corresponding decentralized closed-loop system. Hence, $J_{\text {opt }}$ can alternatively be written as trace $\left(P_{d} X_{0}^{c}\right)$, where $X_{0}^{c}$ is equal to the expected value of the vector $\left[\begin{array}{llll}x(0)^{T} & x^{1}(0)^{T} & \ldots & x^{\nu}(0)^{T}\end{array}\right]^{T}$ times its transpose. Therefore, the discrepancy between $J$ and $J_{o p t}$ can be obtained as follows:

$$
J_{o p t}-J=\operatorname{trace}\left(P_{d} \Pi\right)
$$

where

$\Pi=\left[\begin{array}{cccc}0 & \operatorname{cov}\left(X_{\mu}, X_{\mu}^{1}\right) & \cdots & \operatorname{cov}\left(X_{\mu}, X_{\mu}^{\nu}\right) \\ \operatorname{cov}\left(X_{\mu}^{1}, X_{\mu}\right) & \operatorname{cov}\left(X_{\mu}^{1}, X_{\mu}^{1}\right) & \cdots & \operatorname{cov}\left(X_{\mu}^{1}, X_{\mu}^{\nu}\right) \\ \vdots & \vdots & \ddots & \vdots \\ \operatorname{cov}\left(X_{\mu}^{\nu}, X_{\mu}\right) & \operatorname{cov}\left(X_{\mu}^{\nu}, X_{\mu}^{1}\right) & \cdots & \operatorname{cov}\left(X_{\mu}^{\nu}, X_{\mu}^{\nu}\right)\end{array}\right]$

and $\operatorname{cov}\left(\pi_{1}, \pi_{2}\right)=\mathcal{E}\left\{\pi_{1} \pi_{2}^{T}\right\}-\mathcal{E}\left\{\pi_{1}\right\} \mathcal{E}\left\{\pi_{2}^{T}\right\}$ for any arbitrary column vectors $\pi_{1}$ and $\pi_{2}$.

Remark 4: One can use the equation (39) to find out how close the decentralized performance index $J$ is to the optimal centralized counterpart $J_{o p t}$. In addition, it can be deduced from (39) that the more the initial state $x(0)$ tends to be deterministic, the closer $J$ becomes to $J_{o p t}$, and in the case of a deterministic initial state, $J$ is equal to $J_{o p t}$. This observation along with the result of Theorem 4 confirm that $\tilde{K}_{d}$ is a solution of Problem 1.

\section{NUMERICAL EXAMPLE}

Consider a system $\mathcal{S}$ consisting of two interconnected subsystems with the following state-space representation for its first subsystem $S_{1}$ :

$$
\begin{aligned}
& \dot{x}_{1}(t)=\left[\begin{array}{cc}
1 & -2 \\
2 & 3
\end{array}\right] x_{1}(t)+\left[\begin{array}{l}
1 \\
3
\end{array}\right] u_{1}(t)+E_{1} \omega(t), \\
& y_{1}(t)=\left[\begin{array}{ll}
-1 & 2
\end{array}\right] x_{1}(t)
\end{aligned}
$$

and the following representation for its second subsystem $S_{2}$ :

$$
\begin{aligned}
\dot{x}_{2}(t) & =\left[\begin{array}{ll}
-1 & 2
\end{array}\right] x_{1}(t)-3 x_{2}(t)+5 u_{2}(t)+E_{2} \omega(t), \\
y_{2}(t) & =3 x_{2}(t)
\end{aligned}
$$

where :

- $\omega(t)$ is assumed to be the scalar exponential function $e^{t}$, which represents the structure of the disturbance affecting the input of the system.

- $E_{1}$ and $E_{2}$ are unknown matrices of proper dimensions, which account for the unmeasurable nature of the disturbance in the system.

Assume that the initial state of the system is a random variable with $X_{0}$ (defined in (6)) equal to $I$. It is desired to design a decentralized controller $K_{d}$ to solve Problem 1 under the assumption $Q=R=I$. To this end, an initial centralized controller which can reject the disturbance $\omega(t)$ is to be designed first. This controller is obtained (using the 
method proposed earlier) with the parameters given below:

$$
\begin{aligned}
& \mathcal{A}=\left[\begin{array}{ll}
1 & 0 \\
0 & 1
\end{array}\right], \quad \mathcal{B}_{o}=\left[\begin{array}{ll}
1 & 0 \\
0 & 1
\end{array}\right], \\
& \mathcal{M}_{o}=\left[\begin{array}{cc}
-3.3182 & 0 \\
0 & -1.0393
\end{array}\right] \text {, } \\
& \mathcal{N}_{o}=\left[\begin{array}{ccc}
0.9231 & -4.4856 & 0 \\
0 & 0 & -1.0147
\end{array}\right]
\end{aligned}
$$

Using Algorithm 1 for optimizing the performance of the initial controller, one will arrive at a centralized controller $\tilde{K}_{c}$ described in (31) with the state-space matrices:

$$
\begin{aligned}
\mathcal{B}_{\text {opt }} & =\left[\begin{array}{cc}
3.3253 & 0 \\
0 & 1.6471
\end{array}\right], \\
\mathcal{M}_{\text {opt }} & =\left[\begin{array}{cc}
-0.9348 & -0.0207 \\
0.0988 & -0.5580
\end{array}\right], \\
\mathcal{N}_{\text {opt }} & =\left[\begin{array}{ccc}
0.8214 & -4.2823 & -0.0513 \\
0.0480 & -0.1015 & -0.9764
\end{array}\right]
\end{aligned}
$$

The resultant quadratic performance index $J$ corresponding to the initial controller given to Algorithm 1 and the optimal controller $\tilde{K}_{c}$ are given by 8.6425 and 3.9422 , respectively. This sizable reduction in the cost function points to the effectiveness of Algorithm 1. Now, let decentralize the controller $\tilde{K}_{c}$ using the procedure proposed in Section $\mathrm{V}$, to obtain the local controllers $\tilde{K}_{d_{1}}$ and $\tilde{K}_{d_{2}}$ described by:

$$
\begin{aligned}
& \dot{\eta}_{d_{1}}(t)=\left[\begin{array}{ccc}
-7.8820 & 0.4940 & -2.7898 \\
0 & 1.0000 & 0 \\
4.9412 & 0 & 1.0000
\end{array}\right] \eta_{d_{1}}(t) \\
& +\left[\begin{array}{cc}
-0.7602 & 1.4925 \\
-3.3253 & 6.6506 \\
0 & 0
\end{array}\right] x_{1}(t) \\
& u_{1}(t)=\left[\begin{array}{lll}
-0.0513 & -0.9348 & -0.0207
\end{array}\right] \eta_{d_{1}}(t) \\
& +\left[\begin{array}{ll}
0.8214 & -4.2823
\end{array}\right] x_{1}(t)
\end{aligned}
$$

and:

$$
\begin{aligned}
& \dot{\eta}_{d_{2}}(t)=\left[\begin{array}{cccc}
1.8214 & -6.2823 & -0.9348 & -0.0207 \\
4.4642 & -9.8468 & -2.8043 & -0.0621 \\
-3.3253 & 6.6506 & 1.0000 & 0 \\
0 & 0 & 0 & 1.0000
\end{array}\right] \\
& \times \eta_{d_{2}}(t)+\left[\begin{array}{c}
-0.0513 \\
-0.1539 \\
0 \\
4.9412
\end{array}\right] x_{2}(t) \\
& u_{2}(t)=\left[\begin{array}{llll}
0.0480 & -0.1015 & 0.0988 & -0.5580
\end{array}\right] \eta_{d_{2}}(t) \\
& -0.9764 x_{2}(t)
\end{aligned}
$$

respectively. It is worth mentioning that these local controllers are attained based upon the assumption that every subsystem knows the parameters of the other subsystem, but not necessarily its initial state. To evaluate the performance of the controller $\tilde{K}_{d}$, suppose that the real initial state $x(0)$ is equal to $\left[\begin{array}{lll}1.5 & 1.5 & 1.5\end{array}\right]^{T}$. This represents an inferior scenario in light of the relation $X_{0}=I$ (in fact, it can be easily verified that the initial state given above is noticeably far from its mean). Now, consider two cases as follows:
- Assume that $E_{1}=\left[\begin{array}{ll}1 & 3\end{array}\right]^{T}, E_{2}=[5]$, and that each local controller knows the initial state of the other subsystem with $-100 \%$ error. As a result, the initial states $\eta_{d_{1}}$ and $\eta_{d_{2}}$ are zero vectors. Let the external input $\sin (3 t)$ be applied to the system $\mathcal{S}$, other than the input disturbance. The outputs of the first and the second subsystems of the system $\mathcal{S}$ under the controllers $\tilde{K}_{c}$ and $\tilde{K}_{d}$ are depicted in Figures 1 and 2. As it can be observed, these two controllers perform almost identically such that the discrepancy in their corresponding signals is barely visible (specially in the output $\left.y_{1}(t)\right)$. These figures also illustrate that the disturbance is rejected very quickly and that the steadystate trajectory is reached rather shortly, although the prediction error was significantly large.

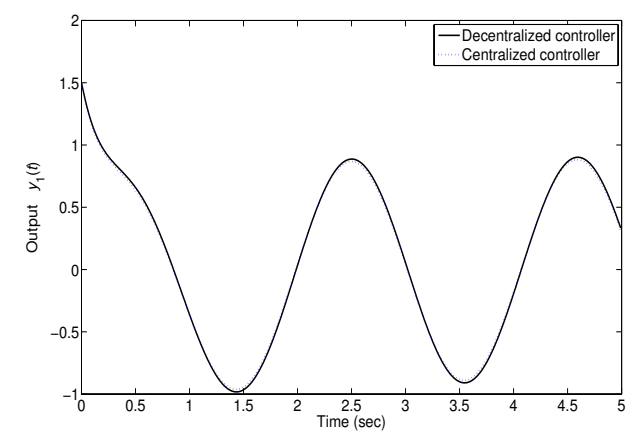

Fig. 1. The output $y_{1}(t)$ in the presence of $-100 \%$ prediction error for the initial state.

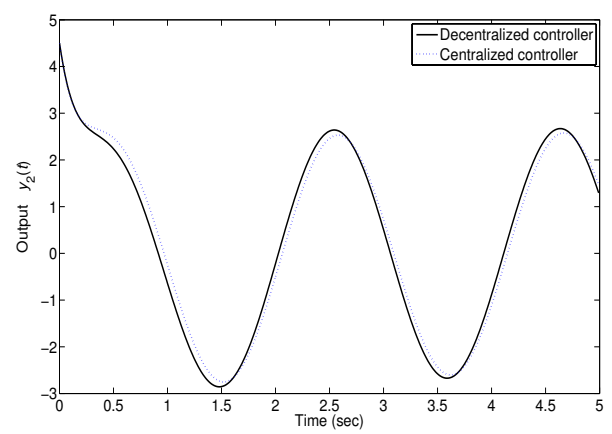

Fig. 2. The output $y_{2}(t)$ in the presence of $-100 \%$ prediction error for the initial state.

- Assume that $E_{1}$ and $E_{2}$ are the same as the ones introduced earlier, and that each local controller knows the initial state of the other subsystem with $5000 \%$ error (i.e. an extremely severe situation is considered here). Hence,

$$
\eta_{d_{1}}=\left[\begin{array}{lll}
75 & 0 & 0
\end{array}\right], \quad \eta_{d_{2}}=\left[\begin{array}{llll}
75 & 75 & 0 & 0
\end{array}\right]
$$

Let the external unbounded input $t \times \sin (t)$ be applied to the system $\mathcal{S}$. The output of the second subsystem of the system $\mathcal{S}$ under the controllers $\tilde{K}_{c}$ and $\tilde{K}_{d}$ is 
depicted in Figure 3 to substantiate how insensitive the decentralized controller $\tilde{K}_{d}$ to prediction error is (due to space restrictions, the output of the first subsystem is not sketched here).

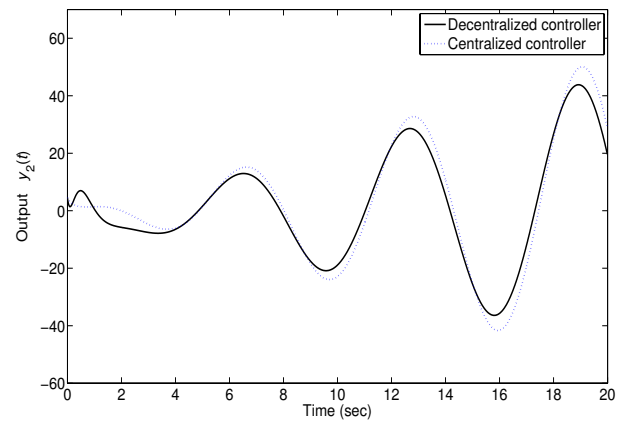

Fig. 3. The output $y_{2}(t)$ in the presence of $5000 \%$ prediction error for the initial state.

\section{CONCLUSIONS}

In this paper, a near-optimal decentralized servomechanism controller is designed for a hierarchical interconnected system. This controller results in a satisfactory performance with respect to a predetermined LQ cost function, and is capable of rejecting unmeasurable external disturbances of known dynamics. The designed controller relies on the information of every individual subsystem about the overall system, and since this information is inexact in practice, a procedure is presented to assess the degradation of the performance of the decentralized control system as a result of the erroneous information. The simulation results demonstrate the effectiveness of the present work.

\section{REFERENCES}

[1] S. Sojoudi, J. Lavaei and A. G. Aghdam, "Optimal information flow structure for control of interconnected systems," in Proc. 2007 American Contrl. Conf., New York, NY, pp. 1508-1513, 2007.

[2] A. G. Aghdam, E. J. Davison and R. B. Arreola, "Structural modification of systems using discretization and generalized sampled-data hold functions," Automatica, vol. 42, no. 11, pp. 1935-1941, Nov. 2006.

[3] G. Inalhan, D. M. Stipanovic and C. J. Tomlin, "Decentralized optimization with application to multiple aircraft coordination," in Proc. 41st IEEE Conf. on Decision and Contrl., Las Vegas, NV, pp. 1147-1155, 2002.

[4] D. M. Stipanovic, G. Inalhan, R. Teo and C. J. Tomlin, "Decentralized overlapping control of a formation of unmanned aerial vehicles," Automatica, vol. 40 , no.8, pp. 1285-1296, Aug. 2004.

[5] S. S. Stankovic, M. J. Stanojevic and D. D. Siljak, "Decentralized overlapping control of a platoon of vehicles," IEEE Trans. Contrl. Sys. Tech., vol. 8, no. 5, pp. 816-832, Sep. 2000.

[6] J. A. Fax and R. M. Murray, "Information flow and cooperative control of vehicle formations," IEEE Trans. Automat. Contrl., vol. 49, no. 9, pp. 1465-1476, Sep. 2004.

[7] J. Lavaei and A. G. Aghdam, "A necessary and sufficient condition for the existence of a LTI stabilizing decentralized overlapping controller," in Proc. 45th IEEE Conf. on Decision and Contrl., San Diego, CA, pp. 6179-6186, 2006.

[8] J. Lavaei and A. G. Aghdam, "High-performance decentralized control design for general interconnected systems with applications in cooperative control," Int. J. Contrl., vol. 80, no. 6, pp. 935-951, 2007.

[9] D. D. Šiljak, Decentralized control of complex systems, Boston: Academic Press, 1991.
[10] E. J. Davison and T. N. Chang, "Decentralized stabilization and pole assignment for general proper systems," IEEE Trans. Automat. Contrl., vol. 35, no. 6, pp. 652-664, Jun. 1990.

[11] J. Lavaei, A. Momeni and A. G. Aghdam, "Spacecraft formation control in deep space with reduced communication requirement," to appear in IEEE Trans. Contrl. Sys. Tech., 2006.

[12] Z. Gong and M. Aldeen, "Stabilization of decentralized control systems," J. Math. Sys. Est. Contrl., vol. 7, no. 1, pp. 1-16, 1997.

[13] J. Lavaei and A. G. Aghdam, "Elimination of fixed modes by means of high-performance constrained periodic control," in Proc. 45th IEEE Conf. on Decision and Contrl., San Diego, CA, pp. 4441-4447, 2006.

[14] J. Lavaei and A. G. Aghdam, "Characterization of decentralized and quotient fixed modes via graph theory," in Proc. 2007 American Contrl. Conf., New York, NY, pp. 790-795, 2007.

[15] J. Leventides and N. Karcanias, "Decentralized dynamic pole assignment with low-order compensators," J. Math. Contrl. Info., available online since Nov. 2006.

[16] S. S. Keerthi and H. S. Phatak, "Regional pole placement of multivariable systems under control structure constraints," IEEE Trans. Automat. Contrl., vol. 40, no. 2, pp. 272-276, Feb. 1995.

[17] M. Rotkowitz and S. Lall, "A characterization of convex problems in decentralized Control," IEEE Trans. Automat. Contrl., vol. 51, no. 2, pp. 274-286, Feb. 2006.

[18] J. Lavaei and A. Aghdam, "Simultaneous LQ control of a set of LTI systems using constrained generalized sampled-data hold functions," Automatica, vol. 43, no. 2, pp. 274-280, Feb. 2007.

[19] E. J. Davison, "The robust decentralized control of a general servomechanism problem," IEEE Trans. Automat. Contrl., vol. 21, no. 1, pp. 14-24, Feb. 1976.

[20] E. J. Davison, "The robust decentralized control of a servomechanism problem for composite systems with input-output interconnections," IEEE Trans. Automat. Contrl., vol. 24, no. 2, pp. 325-327, Apr. 1979.

[21] E. J. Davison, and Ü. Özgüner, "Synthesis of the decentralized robust servomechanism problem using local models," IEEE Trans. Automat. Contrl., vol. 27, no. 3, pp. 583-600, Jun. 1982.

[22] S. V. Savastuk and D. D. Šiljak, "Optimal decentralized control," in Proc. 1994 American Contrl. Conf., Baltimore, MD, pp. 3369-3373, 1994.

[23] D. D. Sourlas and V. Manousiouthakis, "Best achievable decentralized performance," IEEE Trans. Automat. Contrl., vol. 40, no. 11, pp. 18581871, Nov. 1995.

[24] R. Krtolica and D. D. Šiljak, "Suboptimality of decentralized stochastic control and estimation," IEEE Trans. Automat. Contrl., vol. 25, no. 1, pp. 76-83, Feb. 1980.

[25] J. R. Broussard, "An approach to the optimal output feedback initial stabilizing gain problem," in Proc. 29th IEEE Conf. on Decision and Contrl., Honolulu, HI, pp. 2918-2920, 1990.

[26] P. M. Makila and H. T. Toivoneh, "Computational methods for parametric LQ problems- a survey," IEEE Trans. Automat. Contrl., vol. 32, no. 8, pp. 658-671, Aug. 1987.

[27] A. Iftar and Ü. Özgüner, "An optimal control approach to the decentralized robust servomechanism problem," IEEE Trans. Automat. Contrl., vol. 34, no. 12, pp. 1268-1271, Dec. 1989.

[28] R. S. Smith and F. Y. Hadaegh, "Control topologies for deep space formation flying spacecraft," in Proc. 2002 American Contrl. Conf., Anchorage, AK, pp. 2836-2841, 2002.

[29] K. P. Groves, D. O. Sigthorsson, A. Serrani and S. Yurkovich , "Reference command tracking for a linearized model of an air-breathing hypersonic vehicle," in Proc. 2005 AIAA Guid. Navig. Contrl. Conf. Exhibit, San Francisco, CA, 2005.

[30] E. J. Davison, "A generalization of the output control of linear multivariable systems with unmeasurable arbitrary disturbances," IEEE Trans. Automat. Contrl., vol. 20, no. 6, pp. 788-792, Dec. 1975.

[31] J. Lam and Y. Y. Cao, "Simultaneous linear-quadratic optimal control design via static output feedback," Int. J. Robust Nonlinear Contrl., vol. 9, pp. 551-558, 1999.

[32] H. Kwakernaak and R. Sivan, Linear optimal control systems, John Wiley \& sons, 1972. 